\title{
Gestão da informação para a tomada de decisão em uma instituição de ensino superior privada: A experiência das Faculdades Integradas da União Educacional do Planalto Central (FACIPLAC/DF)
}

\author{
Information management for decision making in an institution of private higher \\ edication: the experience of Integrated Colleges of Educational Union of Central Plateau \\ (FACIPLAC/DF)
}

\section{Gleiciane Rosa da Silva \\ Universidade de Brasília.}

\section{RESUMO}

As organizações estão vivenciando a complexidade na gestão devido ao grande volume de informações e exigência por respostas rápidas que demandam tomadas de decisões contínuas e mais assertivas. Assim, o uso de tecnologias e sistemas, apesar de imprescindível para qualquer organização, por si só não garante a obtenção de vantagem competitiva se não houver uma relação direta entre sua adoção e a obtenção de resultados. Nesse contexto, discute-se a seguinte problemática de pesquisa: as decisões adotadas nas Faculdades Integradas da União Educacional do Planalto Central - Faciplac/DF são apoiadas por quais sistemas informacionais? Sob tal enfoque, a justificativa para a realização da pesquisa é a importância que a informação possui para tomada de decisão em uma Instituição de Ensino Superior (IES) as Faculdades Integradas da União Educacional do Planalto Central (Faciplac/DF) a fim de que tais decisões possam tornar a organização mais competitiva no setor em que atua. O objetivo do estudo em questão é analisar a gestão da informação na tomada de decisão na Faculdades Integradas da União Educacional do Planalto Central (Faciplac/DF). A metodologia adotada foi à pesquisa descritiva, com abordagem metodológica quali-quantitativa, ou seja, mista e pesquisa de campo, a ser desenvolvida in loco, utilizando como instrumento entrevista. A técnica de coleta de dados foi realizada pela utilização de um formulário estruturado para investigação, conduzido por entrevistas e observações diretas. Os resultados encontrados apontam para o modelo de gestão da informação na instituição, bem como permite compreender os mecanismos utilizados para tomada de decisão.

PALAVRAS-ChAVE: Gestão da Informação Institucional. Sistema de informação. Tomada de decisão.

\section{Correspondência}

${ }^{1}$ Gleiciane Rosa da Silva

Universidade de Brasília.

Brasília, DF.

Email: gleici.any@hotmail.com

ORCID: http://orcid.org/0000-0001-9210-6467

\begin{abstract}
Organizations are experiencing the complexity of management due to the large volume of information and requirement for rapid responses that require continuous and taken more assertive decisions. Thus, the use of technologies and systems, although essential for any organization by itself does not guarantee the achievement of competitive advantage if there is a direct relationship between its adoption and achieving results. In this context, we discuss the following issues of research: the decisions of the Integrated Faculties of the Central Plateau of the Educational Union - Faciplac / DF are supported by which information systems? Under this approach, the rationale for the research is the importance that information has for decision making in a Higher Education Institution (HEI) - the International College of the Central Plateau of the Educational Union (Faciplac / DF) so that such decisions can make the organization more competitive in the industry in which it operates. The aim of the present study is to analyze the management of information in decisionmaking in the Integrated College of Central Plateau Educational Union (Faciplac / DF). The methodology was the descriptive research with methodological approach qualitative and quantitative, that is, mixed and field research, to be developed in situ, using as an interview tool. The data collection technique was performed by using a form structured to research, conducted interviews and direct observations. The results point to the information management model in the institution and allows us to understand the mechanisms for decision-making.
\end{abstract}

KEYWORDS: Institutional Information Management. Information system. Decision taking.

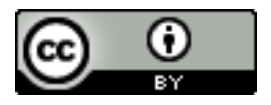

JITA: BH. Information needs and information requirements analysis. 


\section{INTRODUÇÃO}

As organizações estão vivenciando uma nova realidade em que há informação demasiada, o que torna o processo decisório muito mais difícil. Não basta ter informação, é preciso ter a informação correta, no momento adequado, com a qualidade requerida, com o menor custo para que se possa gerar conhecimento e posteriormente inteligência. Para tanto, torna-se imprescindível que as organizações estruturem sistemas de informação para apoiar o processo, e que esses sejam integrados para que se possa obter, armazenar, organizar e distribuir informações relevantes a fim de auxiliar na tomada de decisão.

A informação é um dos elementos mais importantes dentro de uma instituição, constituindo-se como fator que auxilia na estruturação e gestão de seus processos. E, para que tenha êxito, é primordial que a organização compreenda o valor da informação e dos seus sistemas informacionais para tomada de decisão.

Sob tal enfoque, a justificativa para a realização da pesquisa é a importância que a informação possui para tomada de decisão em uma Instituição de Ensino Superior (IES) - as Faculdades Integradas da União Educacional do Planalto Central (Faciplac/DF) a fim de que tais decisões possam tornar a organização mais competitiva no setor em que atua.

O estudo acerca da gestão da informação para tomada de decisão pode contribuir para a área da Ciência da Informação, no sentido de aglutinar maior quantidade de pesquisas sobre o assunto, uma vez que se propõe a estudar elementos de seu interesse, como: informação estratégica, gestão da informação, as atividades de gestão, sistemas de informação, tomada de decisão.

A complexidade dos negócios exige respostas cada vez mais rápidas por parte dos gestores que são responsáveis por tomar decisão. A todo o tempo, tomam-se decisões baseadas em informações que são, também, obtidas por variados sistemas informacionais. Nesse contexto, discute-se a seguinte problemática de pesquisa: as decisões adotadas pelo público-alvo da Faculdades Integradas da União Educacional do Planalto Central Faciplac/DF são apoiadas por quais sistemas informacionais?

Nesse estudo, estabeleceu como objetivo geral identificar os sistemas de informação presentes no processo de tomada de decisão da Administração Básica e Órgãos de Apoio para vislumbrar os elementos de gestão da informação presentes na IES- Faculdades Integradas da União Educacional do Planalto Central - Faciplac/DF. E, para concretizar o objetivo geral, os objetivos específicos foram: identificar os principais modelos da gestão da informação na literatura; descrever os sistemas de informação disponíveis na instituição; identificar quais sistemas de informação são aplicados para a tomada de decisão na instituição; analisar os processos de tomada de decisão da Administração Básica e Órgãos de Apoio. 
A pesquisa limita-se à Comunicação, Comissão própria de Avaliação (CPA), Ouvidoria, Secretaria acadêmica, Coordenação dos cursos de graduação, Coordenação de pós-graduação, iniciação científica e extensão; Núcleo Docente Estruturante (NDE) e acervos. Compondo as esferas: da Administração Básica e Órgãos de Apoio.

O estudo fora assim delimitado, por considerar inviável entrevistar todas as esferas, tanto pelo acesso, quanto pela quantidade de respondentes, levando a necessidade de efetuar um recorte que fosse representativo.

Do universo, participaram como objeto do estudo 15 (quinze) colaboradores do total de 145 (cento e quarenta e cinco) da Administração Básica e Órgãos de Apoio compostos pela: Comunicação, Comissão própria de Avaliação (CPA), Ouvidoria, Secretaria acadêmica, Coordenação dos cursos de graduação, Coordenação de pós-graduação, iniciação científica e extensão; Núcleo Docente Estruturante (NDE) e acervos (administração básica e apoio).

Este artigo encontra-se dividido em cinco seções. A primeira é a introdução composta pela discussão do assunto, justificativa, problema de pesquisa e objetivo do estudo. A segunda seção apresenta a metodologia utilizada para realizar a pesquisa. A terceira seção traz a revisão da literatura com os principais autores. Na quarta seção encontram-se os resultados da pesquisa e a quinta traz as considerações finais.

\section{METODOLOGIA}

Para a realização do estudo em questão foi utilizada a pesquisa descritiva e aplicados os procedimentos de métodos quali-quantitativos, ou seja, método misto.

A coleta de dados (após o pré-teste realizado em dezembro de 2015 para melhor alinhamento da pesquisa) ocorreu por meio do uso das ferramentas: entrevista estruturada e observações, no período de fevereiro e março de 2016, em horários flexíveis, entre 13h às $17 \mathrm{~h}$ e $19 \mathrm{~h}$ às $22: 30 \mathrm{~h}$, de acordo com o horário de trabalho e disponibilidade do público-alvo da pesquisa.

Para a realização do estudo em questão, foi executada uma pesquisa in loco, ou pesquisa de campo com o intuito de atingir os objetivos propostos. E, as técnicas usadas para a coleta de dados foram: a) Entrevista estruturada: conduzida a partir de um roteiro prédeterminado com questões delineadas em formulários; b) Formulários com questões fechadas (que foram formuladas para uso de escala do tipo Likert ${ }^{1}$ ), semi-abertas e abertas; c) Observações.

\footnotetext{
${ }^{1}$ Escala de Likert: Uma escala tipo Likert é composta por um conjunto de frases (itens) em relação a cada uma das quais se pede ao sujeito que está a ser avaliado para manifestar o grau de concordância desde o discordo totalmente (nível 1), até ao concordo totalmente (nível 5, 7 ou 11) (LIMA, 2000)
} 
Os objetivos deste estudo foram alcançados pela convergência entre a pesquisa bibliográfica e pesquisa de campo com o levantamento de informações por intermédio dos instrumentos anteriormente definidos, seguidos de análise das informações quali e quantitativas, com posterior reflexão da pesquisadora.

\section{REVISÃO DA LITERATURA}

\subsection{As Organizações e a Atividade de Gestão}

As organizações são definidas, segundo Maximiano (2009, p. 4), como "sistemas de recursos que procuram realizar algum tipo de objetivo", por isso, conhecer a realidade de uma organização é preponderante para a adoção de sistemas de informação que de fato contribuam para a concretização da sua missão.

Tarapanoff (2001) afirma que, muitos profissionais, partindo de grandes descobertas ao longo da história, como as de Taylor, com a administração científica em 1911, Fayol, com a administração clássica em 1916, passando pela teoria das relações humanas em meados de 1930, a teoria da burocracia com Max Weber e da pesquisa operacional por volta de 1940, do planejamento estratégico nas décadas de 1950 a 1970, teoria comportamental da organização em 1947, da teoria contingencial na década de 70, qualidade total (1980) e da gestão da informação e do conhecimento (1990), passaram por transformações no modo de se organizar e administrar os negócios.

As organizações são tidas como sistemas abertos, em constante mudança, influenciando e sofrendo influências do meio em que estão inseridas (VARAJÃO, 2005).

Um sistema é composto de elementos que se relacionam de modo flexível por meio de entradas (in put), passando por transformações, gerando saídas (out put) e sendo alimentado pelas retroalimentações (feedback).

As organizações são tidas como complexas, necessitando de um entendimento global de sua estrutura de funcionamento, bem como a compreensão de suas relações com a sociedade, economia, política e outros agentes, levando-a a adotar decisões que traduzam sua capacidade de adaptação às mudanças e tendências do mercado.

\subsection{Instituições de Ensino Superior (IES)}

Para Cunha (1980) a educação superior no Brasil desenvolveu-se diferente do restante do continente sul-americano. Com a colonização espanhola criando a primeira universidade em 1538. A história da educação superior no Brasil iniciou-se em 1572 com a concepção dos cursos de Artes e Teologia no Colégio dos Jesuítas da Bahia. Em 1759 ocorreu a expulsão da ordem religiosa, criando oportunidade para a abertura de aulas de matérias isoladas, sendo em 1776, uma faculdade criada no Rio de Janeiro e, em 1798, em Olinda. 
Para Sampaio (2000) é possível afirmar, então, que, os políticos liberais desejavam ver o Estado se retirar progressivamente do campo educacional em nome da liberdade de opinião e crença e consagrar a liberdade do ensino particular primário e médio. Então na República que ocorreu a descentralização do ensino superior para delegação aos governos estaduais, criando então, as instituições privadas.

Fato é que, de acordo com o posicionamento de Trindade (1998), a educação superior no Brasil esteve durante muito tempo, voltada para os filhos das famílias da elite.

Cunha (1980) relata que, após tantos confrontos de interesses, foi no século 20 que fora criada, em 1920, a primeira instituição, a Universidade do Rio de Janeiro. E, a partir desse momento, surgiram outras, tais como a Universidade de Minas Gerais, em 1927, e a Universidade do Rio Grande do Sul, em 1932, apesar de continuarem a trabalhar como englobado de escolas isoladas.

Conforme relata Barreyro (2008), em 1931, no governo de Getúlio Vargas e um ano após a criação do Ministério da Educação, que foram colocadas em prática normas para a organização do ensino superior com a promulgação do Estatuto das Universidades Brasileiras (Decreto $\mathrm{n}^{\circ}$ 19.851/31, vigente até 1961). As universidades poderiam ser mantidas pelo governo federal ou pelos estaduais, portanto oficiais, ou "livres", mantidas por fundações ou associações particulares.

Para Bicalho (2004), é importante ressaltar que, a expansão do ensino superior no Brasil, por intermédio da iniciativa privada, pode ter tido um viés de democratização, pois é indiscutível que ela ampliou o acesso ao nível universitário, no entanto, vale ressaltar que tal expansão é um "fator de mercantilização do ensino", já que muitas instituições oferecem ensino de qualidade duvidosa.

$\mathrm{Na}$ opinião de Picanço (2003), muitos grupos identificaram na educação as possibilidades de um grande e lucrativo mercado. Para esses, fica difícil distinguir conhecimento de mercadoria, pois os estudantes tornaram-se clientes e as instituições constituem-se em empresas com finalidades meramente lucrativas.

No que tange a caracterização das IES, pode-se afirmar que, segundo a Lei 9.394/96, podem estar vinculadas ao sistema federal de ensino ou aos sistemas estaduais e municipais. O sistema federal de ensino é composto, de acordo com o art. 16, Lei 9.394/96 (TANEGUTI, 2013): a) instituições de ensino mantidas pela União; b) instituições de educação superior criadas pela iniciativa privada; e, c) os órgãos federais de educação, subordinadas à União, podendo se organizar como autarquias ou fundações públicas.

\subsection{Gestão da Informação}


Diversas áreas foram aumentando e diversificando suas estratégias para solucionar as dificuldades relativas à organização, ao tratamento e ao uso da informação, iniciando uma preocupação com questões relativas ao planejamento e à implantação de sistemas gerenciais de informação e de conhecimento nas empresas.

Segundo Tarapanoff (2001), o objetivo fundamental da GI é reconhecer e tornar mais forte os recursos informacionais, preparando a organização para um processo de aprendizagem e adaptação inteligente às mudanças do ambiente interno e externo.

A Gestão da Informação pode ser entendida como um conjunto de seis processos diferentes que mantêm relacionamento estreito, cíclico, e que deve ser constantemente alimentado: identificação de necessidades informacionais; aquisição de informação; organização e armazenamento de informação; desenvolvimento de produtos informacionais e serviços; distribuição da informação; e uso da informação (CHOO, 1998).

Taylor e Farrel (1995) ressaltam que o conceito da Gestão da Informação (GI) ainda está em desenvolvimento e comentam três categorias de definições que emergem da literatura: categoria existencial; categoria operacional e categoria Híbrida.

Na visão de Wilson (2002), há o uso ambíguo da expressão Gestão da Informação na literatura de diversas áreas do conhecimento. Para ele, é a aplicação dos princípios da administração à aquisição, organização, controle, disseminação e uso da informação relevante para operação efetiva das organizações de todos os tipos.

Já na opinião de Fairer-Wessels (1997), Gestão da Informação ganha um foco voltado para o planejamento, a organização, a direção e o controle da informação no âmbito da organização.

Uma definição interessante é a proposta por White (1985), na qual a GI permite melhorar a performance da organização por meio da coordenação econômica efetiva da produção, controle, armazenamento, recuperação e disseminação da informação de fontes internas e externas.

Outra contribuição importante que não se pode deixar de abordar é a de Rowley (1998), que considera que a GI gera a efetividade organizacional por intermédio da maximização das capacidades da empresa na lida com demandas de informação de seus ambientes interno e externo em condições dinâmicas e estáveis.

Além de Rowley (1998), Davenport (1998) acrescenta que é necessário pensar estrategicamente acerca da gestão da informação, pois as organizações são dotadas de ambientes informacionais deficientes e recursos informacionais mal-alocados. O uso de estratégias de informação pode ajudar as empresas a se adaptarem às transformações pelo uso da informação mais adequada. 


\subsection{Sistemas de Informação}

Pode-se dizer que sistema de informação, sob uma ótica organizacional, traduz-se como um conjunto de componentes que se relacionam e apresentam características que permitem transferir informações de um ponto a outro, ou seja, do gerador ao usuário, de acordo com regras compatíveis que permitam conexões em diversos âmbitos (AUDY; ANDRADE; CIDRAL, 2005).

Na visão de Oliveira (2009), ao longo do tempo, a teoria de sistemas vem sofrendo transformações e, devido a tal contexto, o moderno enfoque de sistemas, procura desenvolver uma técnica para lidar com a complexidade das organizações, uma visão mais interativa do todo, um estudo das relações entre os elementos componentes em função dos processos e de seus arranjos.

Partindo desse pressuposto, Laudon e Laudon (2001) afirmam que todo sistema de informação é composto por três dimensões: organização, pessoas e tecnologia. Ainda, ressaltam que os sistemas fazem parte das organizações e que os principais elementos são as pessoas, a estrutura, os procedimentos, políticas e cultura.

Oliveira (2009) ressalta que os sistemas são dotados de componentes, tais como: objetivos, entradas, processo de transformação, saídas, controles e avaliações e retroalimentação.

"Sistema é um conjunto de partes interagentes e interdependentes que, conjuntamente, formam um todo unitário com determinado objetivo e efetuam determinada função" (OLIVEIRA, 2009, p. 7).

Araújo Jr. e Alvares (2008) consideram pertinente abordar a necessidade do planejamento do sistema a ser usado pela organização, tendo como ponto de partida o diagnóstico da atual situação, e, a partir dele, selecionar o sistema que melhor se adequa ao contexto estratégico organizacional, englobando a missão, visão e valores da organização.

\subsection{A Tomada de Decisão}

Uma das questões fundamentais, se não a mais importante em uma decisão, é o conflito interno dentro de cada decisor sobre o que se quer fazer e o que precisa ser feito.

Bechara, Damasio, Damasio e Lee (1999) e Bechara, Damasio, Tranel e Damasio (1997) explicam que, regiões do cérebro mais específicas são ativadas para combinar e integrar os sinais que são recebidos durante uma decisão. Para justificar tais alegações, a neurociência evidencia que as regiões do cérebro responsáveis pelas emoções estimulam as decisões baseadas em ações que não contemplam os interesses de longo prazo e que as áreas 
do córtex pré-frontal podem neutralizar os desejos imediatistas e fazer opções de maior valor agregado.

Diante das considerações, as evidências pelo apelo emocional são mais intensas quando se analisa uma alternativa de cada vez, e que decisões mais lógicas e ponderadas serão mais intensas quando avaliadas as alternativas umas contra as outras.

Mediante tais entendimentos, é possível argumentar que a maioria dos profissionais das organizações adotam decisões que ficam aquém do comportamento objetivamente racional. Visando corrigir tais deficiências, Bazerman; Moore (2010) desenvolveu seis estratégias concretas e complementares para adotar melhores decisões, são elas:

a) Utilizar ferramentas que permitam analisar a decisão;

b) Alcançar experiência e conhecimento técnico;

c) Livrar-se das contradições do julgamento;

d) Usar a razão ao relacionar fatos ou situações;

e) Olhar o outro lado, analisar sob a ótica do outro- do agente externo;

f) Compreender as contradições do outro.

Com relação à utilização de ferramentas que permitam analisar a decisão, as técnicas sugeridas exigem que se quantifiquem tanto as preferências quanto o valor que se coloca em cada uma das opções de decisão, usando a lógica do valor esperado que é calculado multiplicando o valor da decisão pela probabilidade. Já para decisões semelhantes que ocorrem repetidamente, uma ferramenta adequada é o modelo linear, calculado por uma fórmula que pesa e soma as variáveis previsoras relevantes a fim de fazer uma previsão quantitativa.

Outra estratégia é alcançar experiência e conhecimento técnico para otimizar a tomada de decisão, além da necessidade de buscar retorno sobre as decisões anteriores. É notório que as experiências da vida ajudam a melhorar várias habilidades, abandonar maus hábitos e aprender de modo responsivo.

Por outro lado, Dawes (1988) afirma que a experiência e o conhecimento podem ser onerosos e que aprender por meio de uma experiência do fracasso, em geral, são aprendizados tidos como "caros".

Na visão de Bazerman, Moore (2010), a mente humana agrupa um poder fenomenal, pois torna possível a realização de atividades que vão desde simples tarefas a complexas e sofisticadas. Ele aborda a anatomia de uma decisão e usa o termo "julgamento" para referirse aos aspectos cognitivos do processo de tomada de decisões, além de analisar as seis etapas que devem ser seguidas ao aplicar um processo racional de tomada de decisão: 
a) Defina o problema: caso não busque especificar e entender bem o problema, pode-se partir para a resolução de outro.

b) Identifique os critérios: para a maioria das decisões, é necessário que se alcance mais de um objetivo.

c) Pondere os critérios: tomadores de decisão racionais devem saber atribuir valores adequados a cada critério.

d) Gere alternativas: é preciso gerar alternativas, mas também é necessário ter cuidado para não perder tempo, nem dinheiro, a fim de originar todas as alternativas possíveis, até mesmo porque não serão escolhidas todas as alternativas.

e) Classifique cada alternativa segundo cada critério: o tomador de decisão deve ser capaz de conseguir avaliar as consequências potenciais da escolha de cada solução, segundo o critério.

f) Identifique a solução ideal: segundo sugestão do autor, é preciso, após ter completado todas as etapas anteriormente sugeridas, multiplicar as classificações da etapa cinco pelo peso de cada critério, somar as classificações ponderadas de todos os critérios para cada alternativa e, por fim, selecionar a solução cuja soma das classificações ponderadas seja a mais alta.

Por meio do modelo anteriormente mencionado, é possível inferir que os decisores definem o problema, identificam critérios, ajuízam com exatidão todos os critérios segundo suas prioridades, conhecem todas as alternativas importantes, avaliam com precisão cada alternativa embasados em cada critério, computando as alternativas com precisão e selecionando aquela que possuir maior valor agregado.

\section{RESULTADOS}

Partindo inicialmente do mapeamento de perfil, foi possível obter os dados, como seguem apresentados no gráfico 1: 
Gráfico 1. Perfil dos participantes (idade, sexo, escolaridade e tempo de empresa)

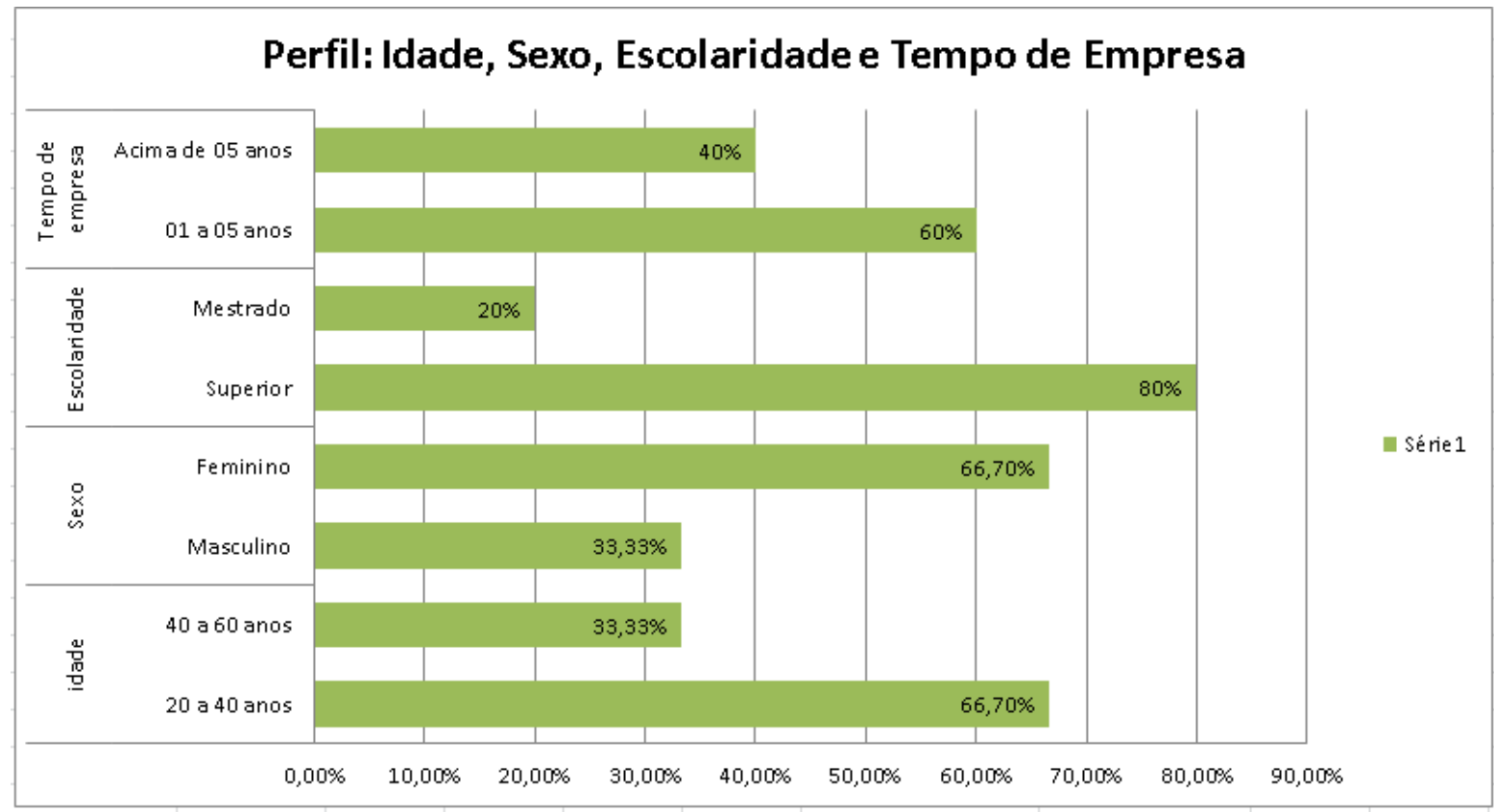

Fonte: Da Autora, 2016.

Como participaram da entrevista 15 (quinze) colaboradores das áreas: Comunicação, Comissão própria de Avaliação (CPA), Ouvidoria, Secretaria acadêmica, Coordenação dos cursos de graduação, Coordenação de pós-graduação, iniciação científica e extensão; Núcleo Docente Estruturante (NDE) e acervos da União Educacional do Planalto Central Faciplac/DF que representam 10\% do universo dessas áreas, pelo mapeamento identifica-se que trata-se de um público jovem, na maioria- feminino, com curso superior e com pouco tempo de trabalho na instituição.

Após o mapeamento do perfil, a entrevista foi conduzida seguindo os passos de construção do formulário:

a) Parte I: informações sobre os processos da instituição;

b) Parte II: informações sobre os veículos de comunicação utilizados na disseminação da informação;

c) Parte III: armazenamento da informação;

d) Parte IV: avaliação geral e Parte V - avaliação da importância da informação e dos sistemas informacionais para a tomada de decisão.

\section{Parte I: Informações sobre os processos da Instituição}

Partindo da proposta de alcançar a propostas complementares ao estudo, foram elaboradas questões sobre o compartilhamento de informações, em que foi possível identificar que algumas áreas compartilham informações com todas as demais: mantenedora,

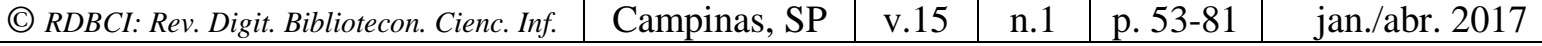


diretoria acadêmica, coordenações, secretaria, biblioteca, recursos humanos, tecnologia da informação, núcleo de desenvolvimento educacional, comunicação, comissão própria de avaliação, colegiados e ouvidoria, entre outras. No entanto, há áreas que o compartilhamento ocorre com um número menor de áreas.

Abaixo segue desenho elaborado pela pesquisadora como uma matriz detalhando a rede de relacionamento entre as áreas, quadro 1:

Quadro 1. Matriz de relacionamento entre as áreas participantes da pesquisa

\begin{tabular}{|c|c|c|c|c|c|c|c|c|c|c|c|c|c|c|c|c|}
\hline \multicolumn{17}{|c|}{ Com que áreas compartilha informações para execução de suas atividades diárias? } \\
\hline Público-alvo & שี & "ॄ] & 可 & 焉 & ๘ & $\frac{8}{4}$ & 兽 & 輎 & 焉 & \% & $\underset{v}{\mathbb{u}}$ & 空 & E & כe & 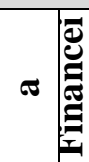 & $\stackrel{0}{2}$ \\
\hline $\begin{array}{l}\text { Núcleo de Iniciação } \\
\text { científica e } \\
\text { extensão/monitorias }\end{array}$ & 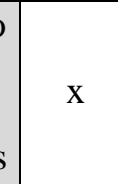 & $\mathrm{X}$ & Y & $X$ & $\mathrm{X}$ & $\mathrm{x}$ & $X$ & & $\mathrm{x}$ & $\mathrm{x}$ & $\mathrm{X}$ & $\mathrm{x}$ & X & $x$ & & \\
\hline NDE & & $\mathrm{x}$ & 2 & $\mathrm{X}$ & & & & & & & & & & & & \\
\hline Coordenações & & $\mathrm{x}$ & Y & $\bar{X}$ & $\mathrm{X}$ & & $\mathrm{X}$ & & & $\mathrm{X}$ & & & & $x$ & & \\
\hline TI & $\mathrm{x}$ & $\mathrm{x}$ & 2 & $\bar{X}$ & $\mathrm{x}$ & $\mathrm{x}$ & $\mathrm{X}$ & & $\mathrm{x}$ & $\mathrm{X}$ & $\mathrm{X}$ & $\mathrm{x}$ & $\mathrm{X}$ & $x$ & & $\mathrm{X}$ \\
\hline Acervo & $\mathrm{x}$ & $\mathrm{X}$ & $\bar{x}$ & $\bar{X}$ & $\mathrm{X}$ & $\mathrm{x}$ & $\mathrm{X}$ & & & & & & $\mathrm{X}$ & $x$ & $\mathrm{x}$ & \\
\hline $\begin{array}{l}\text { Secretaria } \\
\text { Acadêmica }\end{array}$ & $\mathrm{X}$ & $\mathrm{x}$ & 2 & $X$ & $\mathrm{X}$ & & X & & & & & & X & $X$ & & $\mathrm{x}$ \\
\hline Ouvidoria & $\mathrm{x}$ & $\mathrm{x}$ & $\bar{x}$ & $\bar{X}$ & $\mathrm{X}$ & $\mathrm{x}$ & $\mathrm{X}$ & & $\mathrm{x}$ & $\mathrm{X}$ & $\mathrm{X}$ & & $\mathrm{X}$ & $x$ & $\mathrm{x}$ & $\mathrm{X}$ \\
\hline CPA & $x$ & $\mathrm{x}$ & 2 & $\bar{X}$ & $\mathrm{x}$ & & & & $\mathrm{x}$ & $x$ & $\mathrm{x}$ & & $\mathrm{X}$ & $x$ & & $\mathrm{x}$ \\
\hline
\end{tabular}

Fonte: Da Autora, 2016.

Quando questionados se o compartilhamento de informações é suficiente, 26,7\% responderam que sim, enquanto $73,3 \%$ responderam que não é suficiente, e, as principais alegações apresentadas pelos participantes da pesquisa foram:

a) Falta mais investimento em infra-estrutura, como a melhoria dos equipamentos (switch ${ }^{2}$, cabeamento estruturado);

b) Problemas na divulgação de ações do curso que acabam ficando prejudicadas, por não se tornarem conhecidas na organização;

\footnotetext{
${ }^{2}$ Switch: Criado principalmente para resolver os problemas na transmissão de informações. Diferente de seu antecessor (hub), um comutador, como também é chamado, recebe a informação a ser transmitida e a repassa apenas para o destinatário, evitando expô-la a outros computadores.
} 
c) Um grande volume de informações impressas, poucas informações estão disponíveis em formato digital no sistema, fazendo com que as áreas fiquem muito dependentes de outras ou até mesmo as sobrecarregue;

d) A informação é centralizada, quem executa as atividades têm dificuldade para realizalas, por faltar informação; e, e) a informação nem sempre é acessível.

\section{Parte II: Informações sobre os veículos de comunicação utilizados na disseminação da informação}

As primeiras questões foram para o levantamento dos veículos de comunicação mais utilizados para receber e transmitir informações são: reuniões, comunicação verbal e principalmente e-mail. Algumas áreas usam também a intranet, o telefone da instituição. A área da comunicação utiliza além desses: mural, jornal, redes sociais, outdoor, feiras e eventos, stande em shopping e mala direta.

Quando foram questionados acerca desses canais, qual o mais usado para receber e transmitir informações, a resposta foi: e-mail, na grande maioria, seguido de comunicação verbal e aplicativos como whatsapp $^{3}$. A comunicação interna flui entre as áreas, com os subordinados e com discentes.

A comunicação externa não é realizada por todas as áreas, e, as áreas que possuem intercâmbio de informações externas, fazem com: fornecedores de serviços e/ou produtos, representantes comerciais, comunidade, prováveis candidatos a ingressarem na instituição.

E, quanto ao questionamento sobre o que pode ser melhorado em relação aos canais para que as informações possam atingir o público-alvo, foram elencados: a) Gerar protocolos para receber e/ou repassar documentação entre algumas áreas; b) A TI argumentou que as áreas acabam não se utilizando da tecnologia disponível no sistema TOTVS ${ }^{4}$; c) Algumas áreas citaram que deveriam ser mais utilizadas às redes sociais.

\section{Parte III: Armazenamento da Informação}

De acordo com as informações obtidas, nem todos arquivam totalmente todas as informações.

\footnotetext{
${ }^{3}$ Whatsapp: É um software para smartphones utilizado para troca de mensagens de texto instantaneamente, além de vídeos, fotos e áudios através de uma conexão a internet.

${ }^{4}$ A TOTVS é uma empresa brasileira de software, serviços, plataforma e consultoria. Oferece soluções para dez segmentos, sendo elas conduzidas pelos conceitos: tecnologia e ERP.

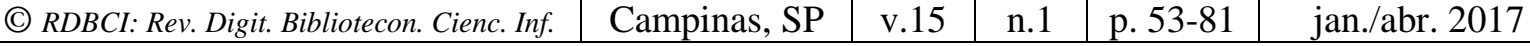


Os meios mais utilizados para tais armazenamentos são: digital (em pastas no sistema TOTVS); pastas suspensas; agenda; e-mail; backup; storage ${ }^{5}$ : citado pela área de TI, como outros meios para armazenar informações.

Um fato importante além do armazenamento é a segurança das informações no sistema. Durante a pesquisa, $80 \%$ dos participantes relataram ter armazenado informações no sistema e posteriormente tais informações se perderam. Desse público, 46,7\% não conseguiu resgatar as informações.

Acerca da memória institucional para buscar informações sobre problemas anteriores idênticos aos atuais para tomada de decisão, 73,3\% concordaram totalmente que houve à necessidade de voltar ao sistema para buscar informações anteriores para tomada de decisão atual.

Uma das principais alegações dos participantes é que o sistema TOTVS utilizado atualmente não permite acessar todas as informações de que necessitam para tomar decisões. Devido ao fato de algumas variáveis não estarem disponíveis ou determinados fatores não estarem visíveis, na visão de Choo (2003) é preciso considerar a necessidade de usar metodologias flexíveis que permitam acessar, representar e conectar a informação.

\section{Parte IV: Avaliação Geral}

Quando questionados sobre quais são as principais dificuldades no processo de acesso/disponibilidade de informação, as respostas foram: o volume de dados é excessivo; as informações estão "presas" em determinadas áreas; os sistemas existentes não fornecem as informações necessárias; existe carência de uma área especializada em gerir informações; e, os canais de comunicação entre o nível da coordenação e direção são insuficientes.

De todas as respostas a que apareceu com maior frequência foi que existe carência de uma área especializada em gerir informações.

Para melhor aprofundar nas questões relativas às principais dificuldades no processo de acesso/disponibilidade de informação, quando questionados se tais dificuldades estão sendo resolvidas, a resposta foi que, para $13,3 \%$ dos participantes, as dificuldades estão sendo resolvidas de forma parcial, porém promissora, enquanto que, $86,70 \%$ responderam que de forma parcial, porém pouco efetiva.

Após a avaliação geral, o último bloco de questões segue abaixo, foi usado para avaliar a importância da informação, de sistemas informacionais no processo de tomada de decisão.

\footnotetext{
${ }^{5}$ Storage: O storage é uma peça altamente redundante e cumpre com louvor a sua missão, que é armazenar os dados da sua empresa com segurança.

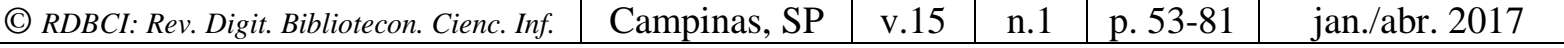




\section{Parte V: Avaliação da importância da informação e dos sistemas informacionais para a tomada de decisão}

Sobre a participação das áreas junto à Diretoria, nas definições estratégicas, táticas e operacionais, segue resultado: Sim: 13,30\%; Não: 40\%; e, Às vezes: 46,70\%.

Com intuito de identificar o modelo de GI aplicado à Faciplac/DF, foi elaborada uma pergunta com todos os modelos citados ao longo desse estudo, abordados pelos autores: Choo, Taylor e Farrel, Wilson, Fair-Wessels, White, Rowley e Davenport, a fim de identificar qual(is) é(são) aplicados na instituição. Os resultados encontrados foram:

a) Proposta apresentada por Taylor e Farrel (1995): 20\%

b) Proposta apresentada por Fairer-Wessels (1997): 46,70\%

c) Proposta apresentada por Rowley (1998): $20 \%$

d) Proposta apresentada por Davenport (1998): 13,33\%

Houve então visões diversificadas acerca da aplicação de modelos de GI na instituição. A opção que selecionada pelos participantes com maior frequência foi à proposta de Fairer-Wessels (1997) que afirmam que a Gestão da Informação tem um foco voltado para o planejamento, a organização, a direção e o controle da informação no âmbito da organização.

Sobre o fato de haver na instituição um grupo de profissionais responsáveis por gerir as informações, seguem resultados: $70 \%$ argumentaram que não existe, enquanto que; 30\% argumentaram que existe e que as áreas responsáveis por gerir informações são: Diretoria, TI e Comunicação.

Acerca dos suportes tecnológicos para acessar informações, os participantes responderam que utilizam: o próprio celular e internet; computador e internet da instituição; intranet; sistema TOTVS.

Complementando, sobre o fato dos usuários terem recebido treinamento, os resultados apontados foram: receberam treinamento: $33,33 \%$; não receberam treinamento: $66,67 \%$ (relataram ter aprendido com os pares, no dia a dia).

Em relação à tomada de decisão, como cada um possui um perfil, adota decisões segundo suas experiências, vivências, conhecimento ou seguindo o formato cultural da instituição.

Após reunir as informações acerca dos passos adotados para tomada de decisão, foi realizada a simulação do modelo seguido pelos profissionais que participaram da pesquisa, para tomar decisões. Tal modelo segue, conforme figura 1: 


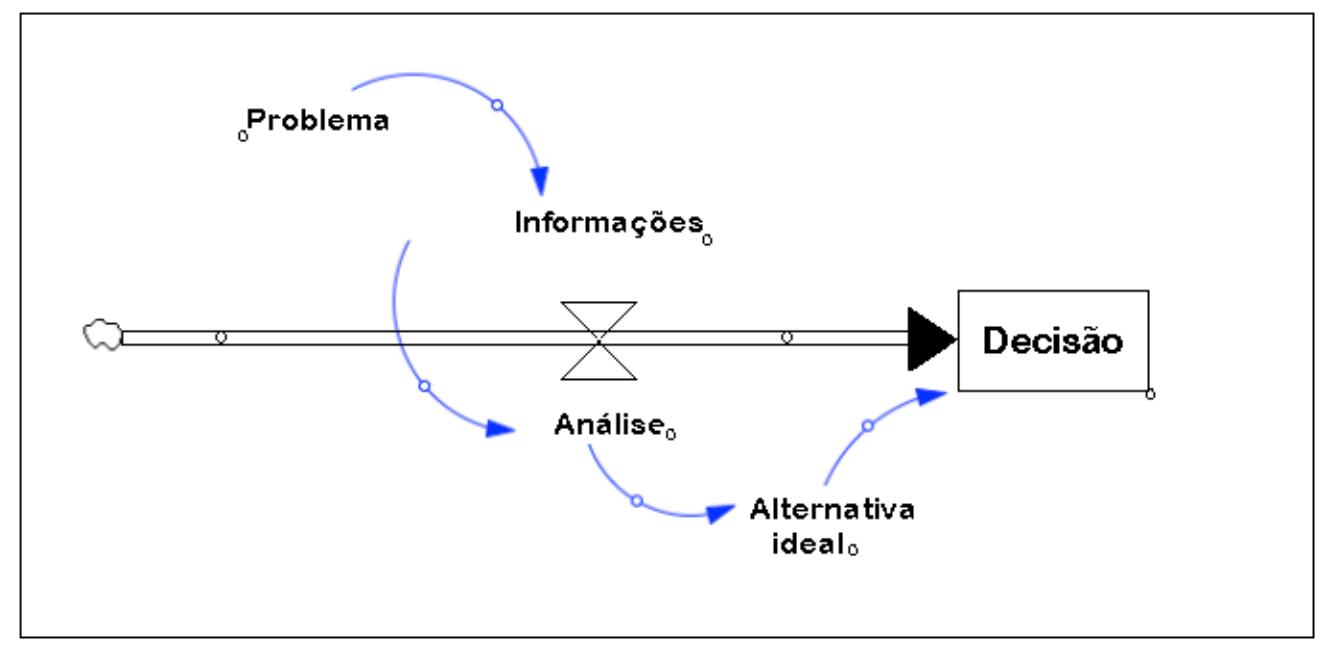

Figura 1. Modelo de Processo de Tomada de Decisão na Faciplac/DF Fonte: Da Autora, 2016.

Baseado nos processos de avaliação de eficiência, efetividade ou eficácia das decisões, $80 \%$ dos participantes alegaram que após a tomada de decisão não utilizam nenhum processo de avaliação, enquanto que, $20 \%$ alegaram que: fazem o acompanhamento das ações; comunicam-se com as pessoas envolvidas nas decisões; e, preparam relatórios e os acompanha.

E, para finalizar a discussão das questões, seguem 04 (quatro) sugestões apontadas pelos participantes, visando melhorar a eficiência, efetividade e eficácia para avaliar as decisões: aumentar o nível de comunicação entre a Direção e as demais áreas; focar no acompanhamento das decisões; estimular o contato pessoal para promover troca de informações sobre decisões adotadas em uma dada área que possam ajudar outras; e, investir em infra-estrutura.

Em síntese, durante a realização desse estudo e ao final, foram identificados: os sistemas de informação; os principais modelos de GI adotados na Faciplac/DF; e, o processo de tomada de decisão, conforme demonstrado na Figura 2:

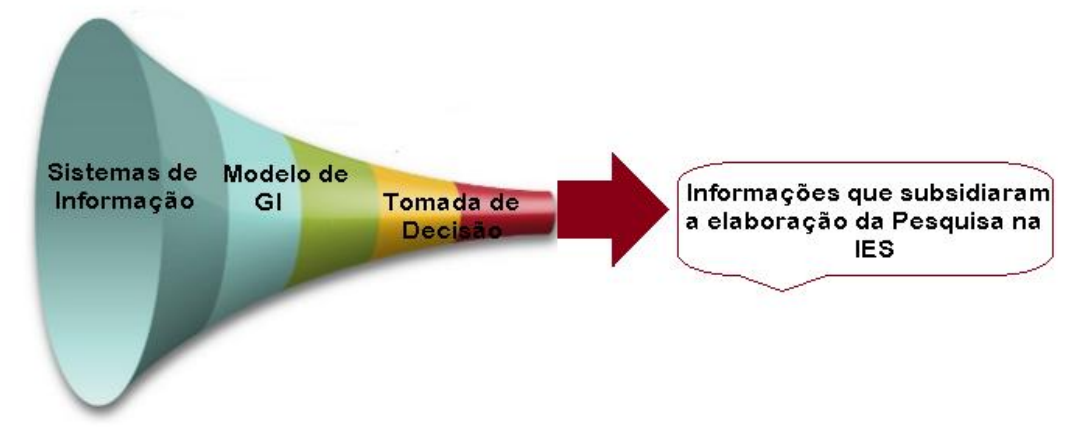

Figura 2. Informações que subsidiaram a elaboração da pesquisa na IES Fonte: Da autora, 2016. 


\section{CONSIDERAÇÕES FINAIS}

A presente pesquisa teve como principal objetivo identificar os sistemas de informação presentes no processo de tomada de decisão da Administração Básica e Órgãos de Apoio para vislumbrar os elementos de gestão da informação presentes na IESFaculdades Integradas da União Educacional do Planalto Central - Faciplac/DF. Para tanto, os esforços foram direcionados a quatro objetivos específicos. A identificação dos principais modelos da gestão da informação resultou no alcance do primeiro objetivo, alcançado pela pesquisa bibliográfica e de campo. O segundo teve como intenção descrever os sistemas de informação disponíveis, o qual foi atingido pela utilização da pesquisa de campo e bibliográfica. Os principais sistemas encontrados, foram: sistema de informação verbal (conversas informais, telefone); sistema de informação escrita (memorando, mural, jornal, email); sistema de informação visual (out-door, standes em shoppings, feiras e eventos); sistema de informação por reuniões; e, sistema de informação tecnológico (sistema informacional da TOTVS, intranet). O terceiro teve por finalidade identificar quais sistemas são usados para tomada de decisão. Acerca da identificação dos sistemas de informação usados para tomada de decisão, os resultados obtidos nessa pesquisa foram alcançados pela adoção da pesquisa de campo - como método de investigação e pela entrevista individual e observações - como fonte de coleta de dados.

Por fim, o quarto objetivo foi analisar os processos de tomada de decisão na Administração Básica e Órgãos de Apoio. Tal objetivo foi alcançado por intermédio da pesquisa de campo, utilizando como fontes de coleta de dados: Entrevista individual (formulário) e Observações.

E, para finalizar as considerações, uma questão percebida durante as observações foi à necessidade de capacitar as áreas nos processos de tomada de decisão, bem como usar tecnologias que favoreçam o acompanhamento e análise das ações, propiciando uma boa gestão da informação. 


\section{LA EXPERIENCIA DE LA UNIÓN DE COLEGIOS INTEGRADO DE LA EDUCACIÓN MESETA CENTRAL (FACIPLAC/DF)}

\section{RESUMEN}

Las organizaciones están experimentando la complejidad de la gestión, debido al gran volumen de información y necesidad de respuestas rápidas que requieren decisiones más asertivas y continuas tomadas. Por lo tanto, el uso de tecnologías y sistemas, aunque esencial para cualquier organización por sí sola no garantiza el logro de una ventaja competitiva si existe una relación directa entre su adopción y el logro de resultados. En este contexto, se discuten los siguientes temas de investigación: las decisiones de las Facultades Integradas de la meseta central de la Unión para la Educación - Faciplac / DF son compatibles con los sistemas de información, que? Bajo este enfoque, la justificación de la investigación es la importancia que tiene la información para la toma de decisiones en una institución de educación superior (IES) - Colegio Internacional de la meseta central de la Unión para la Educación (Faciplac / DF) para que tales decisiones pueden hacer que la organización sea más competitiva en la industria en la que opera. El objetivo del presente estudio es analizar la gestión de la información en la toma de decisiones en el Colegio Integrado de la Unión Central de Educación Meseta (Faciplac / DF). La metodología fue la investigación descriptiva con enfoque metodológico cualitativo y cuantitativo, que la investigación es, mezclado y el campo, a desarrollarse in situ, utilizando como herramienta de entrevista. La técnica de recolección de datos se realizó mediante el uso de una forma estructurada a la investigación, llevó a cabo entrevistas y observaciones directas. Los resultados apuntan al modelo de gestión de la información en la institución y nos permite comprender los mecanismos para la toma de decisiones.

PALABRAS CLAVE: Gestión de información institucional. Sistema de información. Toma de decisiones.

Submetido em: 24/04/2016

Aceito em: 11/07/2016

Publicado em: 03/11/2016

\section{REFERÊNCIAS}

ARAÚJO JR., R. H. de ; ALVARES, L. As necessidades informacionais dos usuários no planejamento e modelagem de sistemas de informação. Revista IberoAmericana de Ciência da Informação, v. 1, p. 201-221, 2008.

AUDY, J. L. N.; ANDRADE, G. K. de; CIDRAL, A. Fundamentos de Sistemas de Informação. Porto Alegre. Bookman, 2005.

BARREYRO, Gladys Beatriz. Mapa do ensino superior privado. Brasília: Instituto Nacional de Estudos e Pesquisas Educacionais Anísio Teixeira, 2008. Disponível em: http://each.uspnet.usp.br/gladysb/Mapa\%20do\%20ensino\%20superior\%20privado.pdf. Acesso em: 12/03/2016.

BAZERMAN, M.H., MOORE, D. Processo decisório. 7. ed. Rio de Janeiro: Elsevier, 2010. 
BECHARA, A.; DAMASIO, H.; DAMASIO, A. R. e LEE, G. P. Different contribuitions of the human amygdala and ventromedial pré-frontal córtex to decision-making. Journal of Neuro-science, 1999.

BICALHO, M. G. P. Ensino superior privado, relação com o saber e reconstrução identitária. 2004. 194 f. Tese (Doutorado em Educação) - Faculdade de Educação, Universidade do Estado de Minas Gerais, Belo Horizonte, 2004.

$\mathrm{CHOO}, \mathrm{C} . \mathrm{W}$. Information management for the Intelligence organization; the art of scanning the environment. 2. ed. Medford, N. J. Information Today, 1998 (ASIS Monograph Series).

CHOO, C. W. A organização do conhecimento: como as organizações usam a informação para criar significado, construir conhecimento e tomar decisões. Tradução de Eliane Rocha. 2. ed. São Paulo: Editora Senac, 2003. 425 p.

CUNHA, L. A. A universidade temporã: o ensino superior da Colônia à era de Vargas. Rio de Janeiro: Civilização Brasileira, 1980.

DAMASIO, H.; TRANEL, D. ; DAMASIO, A. R. Deciding advantageously before knowing the advantageous strategy. Science, 1997.

DAVENPORT, Thomas H. Ecologia da Informação: porque só a tecnologia não basta para o sucesso na era da informação/Thomas H. Davenport, Laurecnce Prusak, tradução Bernadette Siqueira Abrão. São Paulo: Futura, 1998.

DAWES, R. M. Rational choice in an uncertain world. Fort worth, TX: Harcourt Brace, 1998.

FAIRER-WESSELS, F. A. Information management education: towards a holistic perspective. South African Journal of Library and Information Science, v. 65, n. 2, p. 93 $102,1997$.

LAUDON, K.; LAUDON, J. P. Gerenciamento de sistemas de informação. 3. ed. Rio de Janeiro: Editora S.A, 2001.

MAXIMIANO, A. C. A. Teoria geral da administração. São Paulo: Atlas: 2009.

OLIVEIRA, D. de P. R. Sistemas de informações gerenciais: estratégias, táticas operacionais. 13. ed. São Paulo: Editora Atlas, 2009.

PICANÇO, A. A. Educação superior para professores em exercício: formando ou improvisando? In: REUNIÃO DA ASSOCIAÇÃO NACIONAL DE PESQUISA E PÓSGRADUAÇÃO EM EDUCAÇÃO, 26., Caxambu, 2003. Anais. CD-ROM.

ROWLEY, J. Towards a framework for information management. International Journal of Information Management, v. 8, n. 5, p. 359-369, 1998.

SAMPAIO, H. O ensino superior no Brasil: o setor privado. São Paulo: Hucitec, Fapesp, 2000.

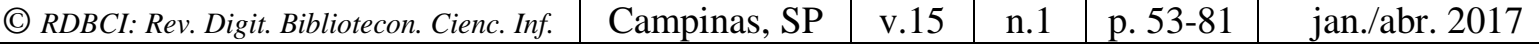


TANEGUTI, Luiza Yoko. Relatório técnico contendo estudo sobre a atual relação oferta/demanda de cursos de graduação no Brasil, como subsídio ao Conselho Nacional de Educação para a formulação de políticas públicas que possibilitem a melhor distribuição da oferta de vagas no ensino superior de graduação. PROJETO CNE/UNESCO 914BRZ1136.3 "Desenvolvimento, aprimoramento e consolidação de uma educação nacional de qualidade". MINISTÉRIO DA EDUCAÇÃO CONSELHO NACIONAL DE EDUCAÇAO CÂMARA DE EDUCAÇAO SUPERIOR. 2013. Disponível em:

http://portal.mec.gov.br/index.php?option=com_docman\&view=download\&alias $=13948$ produto-2-oferta-demanda-educ-superior-pdf-pdf\&category_slug=setembro-2013pdf\&Itemid=30192. Acesso em: 20/03/2016, 11:59h.

TARAPANOFF, K. (Org.). Inteligência organizacional e competitiva. Brasília: Editora Universidade de Brasília, 2001.

TAYLOR, A.; FARREL, S. Information management for business. Metuchen, London: Aslib, 1995. 169 p.

TOTVS. Institucional. Disponível em: https://www.totvs.com/a-totvs. Acesso em: 28/03/2016.

TRINDADE, H. Universidade em perspectiva: sociedade, conhecimento e poder. In: REUNIÃO ANUAL DA ANPED, 21., 1998. Trabalho apresentado. Política de Educação Superior. Caxambu, set. 1998. Disponível em: http://www.anped.org.br Acesso em:20/03/2016, 11:55h.

VARAJÃO, J. E. Q. A arquitetura da gestão de sistemas de informação, FCA, 2005.

WHITE, M. Intelligence management. In: CRONIN, B. (Ed.). Information management: from strategies to action. Londres: Aslib, 1985.

WILSON, T. D. Information management. In: FEATHER, J.; STURGES, P. (Ed.). International Encyclopedia of Information and Library Science. Londres: Rout leg, 2002.

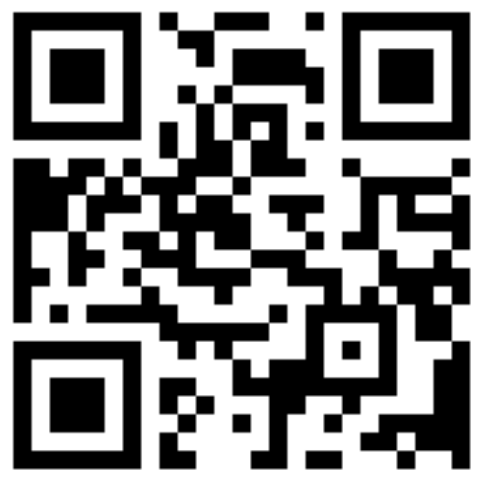




\section{APÊNDICE A}

\section{QUESTIONÁRIO}

Caro(a) senhor(a), participante do estudo,

Este levantamento faz parte da pesquisa de mestrado realizada no Programa de Pós-Graduação em Ciência da Informação da Universidade de Brasília, com o apoio do das Faculdades Integradas da União Educacional do Planalto Central (Faciplac).

Cabe esclarecer que, por meio de Entrevistas, ele será respondido.

O título da pesquisa é "Gestão da Informação para a Tomada de Decisão em uma Instituição de Ensino Superior Privada - A Experiência da Faculdades Integradas da União Educacional do Planalto Central (Faciplac/Df)" tomando como ponto de partida diversos modelos, entre eles, o Modelo de Choo (1998) e tem como principal objetivo identificar como ocorre o processo de gestão da informação e quais sistemas de informação são aplicados para a tomada de decisão na Administração Básica e Órgãos de Apoio.

Para alcançar o objetivo a pesquisa prevê como instrumento: entrevista estruturada por meio de formulário.

É importante ressaltar que os dados serão usados única e exclusivamente para fins acadêmicos e científicos.

Sua contribuição será muito importante.

Agradeço desde já a presteza em colaborar com a pesquisa.

Gleiciane Rosa da Silva

Professora da Faciplac/DF. Matrícula: 100848

Mestranda em Ciência da Informação pela UnB.

Especialista em Engenharia de Produção (UniEvangélica).

Pós Graduada em Docência do Ensino Profissionalizante (SENAC/Goiás)

Graduada em Administração.

Para mais informações:

Currículo Lattes:

$<$ https://wwws.cnpq.br/cvlattesweb/PKG_MENU.menu?f_cod=5DC1EA3E6482EA4FB51E9DEA8C 90A6FB>.

Correio $\quad$ eletrônico: $\quad$ gleiciane.silva@faciplac.edu.br; $\quad$ gleici.any@hotmail.com; gleicianerosasilva@gmail.com

1. Dados de identificação do respondente:

Nome:

Idade:_ Sexo: ( ) M ( ) F Escolaridade:

Cargo ocupado na empresa:

Anos de trabalho na empresa:

\section{I - Informações sobre os processos da Instituição}

\begin{tabular}{|c|c|c|c|c|c|}
\hline (C) RDBCI: Rev. Digit. Bibliotecon. Cienc. Inf. & Campinas, SP & $\mathrm{v} .15$ & n. 1 & p. 53-81 & jan./abr. 2017 \\
\hline
\end{tabular}


1) Há compartilhamento de informações da sua área com demais áreas.

\begin{tabular}{|c|c|c|c|c|}
\hline $\begin{array}{c}\text { Discordo } \\
\text { Totalmente }\end{array}$ & $\begin{array}{c}\text { Discordo } \\
\text { Parcialmente }\end{array}$ & $\begin{array}{c}\text { Não concordo, } \\
\text { nem discordo }\end{array}$ & $\begin{array}{c}\text { Concordo } \\
\text { parcialmente }\end{array}$ & $\begin{array}{c}\text { Concordo } \\
\text { totalmente }\end{array}$ \\
\hline 1 & 2 & 3 & 4 & 5 \\
() & $(\quad)$ & $($ ) & $($ ) & $($ ) \\
\hline
\end{tabular}

2) Com quais áreas compartilha informações para execução de suas atividades diárias?
( ) Mantenedora
( ) Diretoria acadêmica
( ) Coordenações

( ) Secretaria ( ) Biblioteca

( ) $\mathrm{RH}$

( ) TI

( ) $\mathrm{NDE}$
( ) Comunicação
( ) $\mathrm{CPA}$
( ) Colegiados
( ) Ouvidoria

De todas as áreas cite as 03 (três) principais áreas de compartilhamento informacional:

3) Esse compartilhamento de informações é suficiente? ( ) Sim （ ) Não

3.1.1) Caso não seja, relate problemas/dificuldades enfrentadas:

4) Há compartilhamento de informações da sua coordenação para seus subordinados.

\begin{tabular}{|c|c|c|c|c|}
\hline $\begin{array}{c}\text { Discordo } \\
\text { Totalmente }\end{array}$ & $\begin{array}{c}\text { Discordo } \\
\text { Parcialmente }\end{array}$ & $\begin{array}{c}\text { Não concordo, } \\
\text { nem discordo }\end{array}$ & $\begin{array}{c}\text { Concordo } \\
\text { parcialmente }\end{array}$ & $\begin{array}{c}\text { Concordo } \\
\text { totalmente }\end{array}$ \\
\hline 1 & 2 & 3 & 4 & 5 \\
() & $(\quad)$ & $(\quad)$ & $($ ) & $($ ) \\
\hline
\end{tabular}

4.1) Esse compartilhamento é suficiente para execução das atividades entre a coordenação e seus subordinados.

\begin{tabular}{|c|c|c|c|c|}
\hline $\begin{array}{c}\text { Discordo } \\
\text { Totalmente }\end{array}$ & $\begin{array}{c}\text { Discordo } \\
\text { Parcialmente }\end{array}$ & $\begin{array}{c}\text { Não concordo, } \\
\text { nem discordo }\end{array}$ & $\begin{array}{c}\text { Concordo } \\
\text { parcialmente }\end{array}$ & $\begin{array}{c}\text { Concordo } \\
\text { totalmente }\end{array}$ \\
\hline 1 & 2 & 3 & 4 & 5 \\
$(\quad)$ & $(\quad)$ & () & $(~)$ & () \\
\hline
\end{tabular}

4.1.1) Relate problemas/dificuldades enfrentadas ou marque ( ) Não aplicável:

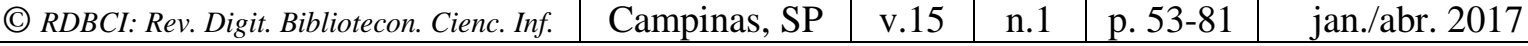


5) O Sr.(a) depende mais de informações internas para suas decisões do que informações externas.

\begin{tabular}{|c|c|c|c|c|}
\hline $\begin{array}{c}\text { Discordo } \\
\text { Totalmente }\end{array}$ & $\begin{array}{c}\text { Discordo } \\
\text { Parcialmente }\end{array}$ & $\begin{array}{c}\text { Não concordo, } \\
\text { nem discordo }\end{array}$ & $\begin{array}{c}\text { Concordo } \\
\text { parcialmente }\end{array}$ & $\begin{array}{c}\text { Concordo } \\
\text { totalmente }\end{array}$ \\
\hline 1 & 2 & 3 & 4 & 5 \\
() & () & () & () & () \\
\hline
\end{tabular}

5.1) Sr.(a) se comunica com o público-alvo externo? ( ) Sim （ ） Não

5.2) Quem é esse público?

6) A divulgação de informações internas na empresa atinge os objetivos, pois alcança todos que dela necessitam.

\begin{tabular}{|c|c|c|c|c|}
\hline $\begin{array}{c}\text { Discordo } \\
\text { Totalmente }\end{array}$ & $\begin{array}{c}\text { Discordo } \\
\text { Parcialmente }\end{array}$ & $\begin{array}{c}\text { Não concordo, } \\
\text { nem discordo }\end{array}$ & $\begin{array}{c}\text { Concordo } \\
\text { parcialmente }\end{array}$ & $\begin{array}{c}\text { Concordo } \\
\text { totalmente }\end{array}$ \\
\hline 1 & 2 & 3 & 4 & 5 \\
() & $(\quad)$ & () & () & $($ ) \\
\hline
\end{tabular}

6.1) Comentários acerca de questões que possam ser melhoradas:

7) Quando o Sr.(a) recebe alguma informação (da diretoria, secretaria ou demais áreas) precisa tratá-las/ajustá-las antes de repassá-las.

\begin{tabular}{|c|c|c|c|c|}
\hline $\begin{array}{l}\text { Discordo } \\
\text { Totalmente }\end{array}$ & $\begin{array}{c}\text { Discordo } \\
\text { Parcialmente }\end{array}$ & $\begin{array}{c}\text { Não concordo, } \\
\text { nem discordo }\end{array}$ & $\begin{array}{c}\text { Concordo } \\
\text { parcialmente }\end{array}$ & $\begin{array}{c}\text { Concordo } \\
\text { totalmente }\end{array}$ \\
\hline 1 & 2 & 3 & 4 & 5 \\
() & () & $(\quad)$ & () & () \\
\hline
\end{tabular}

7.1) Cite o tipo de tratativa/ajuste que são necessários:

8) O Sr.(a) precisa com frequência ir atrás da informação, porque ela, geralmente, não chega em tempo hábil para suas decisões.

\begin{tabular}{|c|c|c|c|c|}
\hline $\begin{array}{l}\text { Discordo } \\
\text { Totalmente }\end{array}$ & $\begin{array}{c}\text { Discordo } \\
\text { Parcialmente }\end{array}$ & $\begin{array}{c}\text { Não concordo, } \\
\text { nem discordo }\end{array}$ & $\begin{array}{c}\text { Concordo } \\
\text { parcialmente }\end{array}$ & $\begin{array}{c}\text { Concordo } \\
\text { totalmente }\end{array}$ \\
\hline 1 & 2 & 3 & 4 & 5 \\
\hline
\end{tabular}

\begin{tabular}{l|l|l|l|l|l}
\hline (C) RDBCI: Rev. Digit. Bibliotecon. Cienc. Inf. & Campinas, SP & v.15 & n.1 & p. 53-81 & jan./abr. 2017
\end{tabular} 


\begin{tabular}{|l|l|l|l|l|}
\hline() & $($ ) & $($ ) & $($ ) & $($ ) \\
\hline
\end{tabular}

9) As informações para sua tomada de decisão são fáceis de serem obtidas e estão sempre disponíveis.

\begin{tabular}{|c|c|c|c|c|}
\hline $\begin{array}{l}\text { Discordo } \\
\text { Totalmente }\end{array}$ & $\begin{array}{c}\text { Discordo } \\
\text { Parcialmente }\end{array}$ & $\begin{array}{c}\text { Não concordo, nem } \\
\text { discordo }\end{array}$ & $\begin{array}{c}\text { Concordo } \\
\text { parcialmente }\end{array}$ & $\begin{array}{c}\text { Concordo } \\
\text { totalmente }\end{array}$ \\
\hline 1 & 2 & 3 & 4 & 5 \\
$(\quad)$ & $(\quad)$ & $($ ) & $(~)$ & $($ ) \\
\hline
\end{tabular}

9.1) Quais informações você necessita e não estão disponíveis?

10) O Sr.(a) precisa com frequência consultar a veracidade das informações recebidas de outras áreas antes de utilizá-las ou repassá-las.

\begin{tabular}{|c|c|c|c|c|}
\hline $\begin{array}{l}\text { Discordo } \\
\text { Totalmente }\end{array}$ & $\begin{array}{c}\text { Discordo } \\
\text { Parcialmente }\end{array}$ & $\begin{array}{c}\text { Não concordo, } \\
\text { nem discordo }\end{array}$ & $\begin{array}{c}\text { Concordo } \\
\text { parcialmente }\end{array}$ & $\begin{array}{c}\text { Concordo } \\
\text { totalmente }\end{array}$ \\
\hline $\begin{array}{c}1 \\
(()\end{array}$ & 2 & 3 & 4 & 5 \\
$(\quad)$ & $(~)$ & () & () \\
\hline
\end{tabular}

\section{II - Informações sobre os veículos de comunicação utilizados na disseminação da informação:}

1) Por meio de quais canais o Sr.(a) recebe informações:
( ) Reuniões ( ) Intranet
( ) Comunicação verbal
( ) Memorando
( ) e-mail
( ) Sistema
( ) Outros:

De todos os canais, o Sr.(a) recebe mais informações de qual?

2) Por meio de quais canais divulga/repassa as informações:
( ) Reuniões
( ) Mural
( ) Intranet
( ) Comunicação verbal
( ) Memorando
( ) Jornal
( ) e-mail
( ) Sistema
( ) Outros:

De todos os canais, divulga mais informações por meio de qual? 
3) Os canais usados para o Sr.(a) divulgar/repassar as informações internas atinge o públicoalvo.

\begin{tabular}{|c|c|c|c|c|}
\hline $\begin{array}{c}\text { Discordo } \\
\text { Totalmente }\end{array}$ & $\begin{array}{c}\text { Discordo } \\
\text { Parcialmente }\end{array}$ & $\begin{array}{c}\text { Não concordo, } \\
\text { nem discordo }\end{array}$ & $\begin{array}{c}\text { Concordo } \\
\text { parcialmente }\end{array}$ & $\begin{array}{c}\text { Concordo } \\
\text { totalmente }\end{array}$ \\
\hline 1 & 2 & 3 & 4 & 5 \\
$(\quad)$ & $(\quad)$ & $(\quad)$ & $($ ) & $($ ) \\
\hline
\end{tabular}

3.1) O que pode ser melhorado?

4) Os canais usados para divulgar as informações externas atinge o público-alvo externo.

\begin{tabular}{|c|c|c|c|c|}
\hline $\begin{array}{c}\text { Discordo } \\
\text { Totalmente }\end{array}$ & $\begin{array}{c}\text { Discordo } \\
\text { Parcialmente }\end{array}$ & $\begin{array}{c}\text { Não concordo, } \\
\text { nem discordo }\end{array}$ & $\begin{array}{c}\text { Concordo } \\
\text { parcialmente }\end{array}$ & $\begin{array}{c}\text { Concordo } \\
\text { totalmente }\end{array}$ \\
\hline 1 & 2 & 3 & 4 & 5 \\
() & () & () & () & () \\
\hline
\end{tabular}

4.1) O que pode ser melhorado?

4.1.1) Canais usados externamente:
( ) Jornal televisivo
( ) Outdoor ( ) Facebook/redes sociais
( ) Feiras e eventos
( ) Stande em shoppings
( ) mala direta
( ) Outros:

\section{III - Armazenamento da Informação:}

1) O Sr.(a) arquiva ou guarda as informações que você recebe ou transmite, para posterior consulta ou comprovação?

\begin{tabular}{|c|c|c|c|c|}
\hline $\begin{array}{c}\text { Discordo } \\
\text { Totalmente }\end{array}$ & $\begin{array}{c}\text { Discordo } \\
\text { Parcialmente }\end{array}$ & $\begin{array}{c}\text { Não concordo, } \\
\text { nem discordo }\end{array}$ & $\begin{array}{c}\text { Concordo } \\
\text { parcialmente }\end{array}$ & $\begin{array}{c}\text { Concordo } \\
\text { totalmente }\end{array}$ \\
\hline 1 & 2 & 3 & 4 & 5 \\
() & () & $(~)$ & $(~)$ & () \\
\hline
\end{tabular}

2) Quais são os meios usados para armazenar informações?
( ) Digital
( ) Backup
( ) Pastas suspensas

( ) Sistema Faciplac ( ) HD externo

( ) e-mail ( ) Outros:

3) O Sr.(a) já armazenou informações no sistema e posteriormente elas desapareceram.

\begin{tabular}{|c|c|c|c|c|}
\hline $\begin{array}{c}\text { Discordo } \\
\text { Totalmente }\end{array}$ & $\begin{array}{c}\text { Discordo } \\
\text { Parcialmente }\end{array}$ & $\begin{array}{l}\text { Não concordo, } \\
\text { nem discordo }\end{array}$ & $\begin{array}{c}\text { Concordo } \\
\text { parcialmente }\end{array}$ & $\begin{array}{l}\text { Concordo } \\
\text { totalmente }\end{array}$ \\
\hline
\end{tabular}

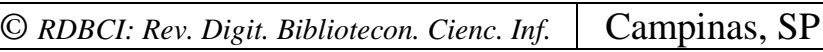




\begin{tabular}{|c|c|c|c|c|}
\hline 1 & 2 & 3 & 4 & 5 \\
() & () & $(~)$ & $(~)$ & $(~)$ \\
\hline
\end{tabular}

4) O Sr.(a) conseguiu resgatar informações que haviam desaparecido do sistema.

\begin{tabular}{|c|c|c|c|c|}
\hline $\begin{array}{c}\text { Discordo } \\
\text { Totalmente }\end{array}$ & $\begin{array}{c}\text { Discordo } \\
\text { Parcialmente }\end{array}$ & $\begin{array}{c}\text { Não concordo, } \\
\text { nem discordo }\end{array}$ & $\begin{array}{c}\text { Concordo } \\
\text { parcialmente }\end{array}$ & $\begin{array}{c}\text { Concordo } \\
\text { totalmente }\end{array}$ \\
\hline 1 & 2 & 3 & 4 & 5 \\
() & () & () & () & $($ ) \\
\hline
\end{tabular}

5) O Sr.(a) já precisou acessar o sistema para buscar informações em relatórios acerca de problemas anteriores idênticos aos atuais para tomada de decisão.

\begin{tabular}{|c|c|c|c|c|}
\hline $\begin{array}{c}\text { Discordo } \\
\text { Totalmente }\end{array}$ & $\begin{array}{c}\text { Discordo } \\
\text { Parcialmente }\end{array}$ & $\begin{array}{c}\text { Não concordo, } \\
\text { nem discordo }\end{array}$ & $\begin{array}{c}\text { Concordo } \\
\text { parcialmente }\end{array}$ & $\begin{array}{c}\text { Concordo } \\
\text { totalmente }\end{array}$ \\
\hline 1 & 2 & 3 & 4 & 5 \\
() & () & $(\quad)$ & $($ ) & $($ ) \\
\hline
\end{tabular}

6. O sistema da Faciplacf utilizado hoje permite que o Sr.(a) acesse todas as informações de que necessita para tomada de decisão?

( ) Sim ( ) Não.

Quais informações o Sr.(a) acessa via sistema?

Quais informações o Sr.(a) precisaria acessar via sistema?

\section{PARTE IV - AVALIAÇÃO GERAL}

1. Em sua opinião, essas dificuldades estão sendo corrigidas?

Sim, sempre;

De forma parcial, porém promissora;

$\square$ De forma parcial e pouco efetiva (algumas sim, outras não)

$\square$ Não.

\section{APÊNDICE B}

PARTE V - Avaliação da importância da informação e dos sistemas informacionais para a tomada de decisão 
A entrevista a ser realizada com o público-alvo da pesquisa seguirá o roteiro abaixo e será usada como ferramenta de avaliação da informação e de sistemas no processo de tomada de decisão na empresa.

1) Sobre quais os processos o Sr. (a) toma decisões diariamente?
( ) contratação
( ) desligamento
( ) divulgação do curso

( ) atividades operacionais

( ) treinamento

( ) recebimento e repasse de informações

( ) Outros:

1.1) Quais decisões mais comuns, precisa adotar em seu dia-a-dia?

2) Das informações que recebe para tomar decisões, o maior volume está relacionado a quê?
( ) problemas de grade de aluno
( ) demandas para divulgar o curso

( ) problemas com carga horária de professores

( ) Outros:

3) O Sr.(a) participa junto à Diretoria, de definições estratégicas, táticas ou operacionais?
( ) $\operatorname{Sim}$
( ) Não
( ) Às vezes.

Quais:

4) Cite fatores a serem melhorados para o processo anterior.

5) Em sua opinião, Gestão da Informação (GI), na Faciplac/DF pode ser identificada por meio de uma rede de processos que:

a) ( ) que permitem obter, gerar, organizar, distribuir e utilizar informação. Os processos são definidos pela: identificação das necessidades de informação; coleta de informação; organização e armazenamento de informação; disseminação da informação (desenvolvimento de produtos e serviços de informação); uso da informação e comportamento adaptativo (Choo, 1998). 
b) ( ) partem primeiro de definições voltadas para as origens e os elementos da Gestão da Informação; segundo, na gestão de recursos de informação para alcançar os objetivos corporativos estratégicos e, por fim, definir as respostas organizacionais para os desenvolvimentos em tecnologias de informação (Tayloy e Farrel, 1995).

c) ( ) se relacionam com a aplicação dos princípios da administração voltados à aquisição, organização, controle, disseminação e uso da informação relevante para operação efetiva das organizações de todos os tipos (Wilson, 2000).

d) ( ) que ganha um foco voltado para o planejamento, a organização, a direção e o controle da informação no âmbito da organização (Fairer-Wessels, 1997).

e) ( ) permite melhorar a performance da organização por meio da coordenação econômica efetiva da produção, controle, armazenamento, recuperação e disseminação da informação de fontes internas e externas (White, 1985).

f) ( ) permite ter duas dimensões: 1. Voltada à gestão do processo informacional e outra 2. voltada a gestão de recursos de dados (Rowley, 1998).

g) ( ) estruturado de ações que abarcam a maneira como as organizações: adquirem, entregam e utilizam a informação e o conhecimento (Davenport, 1998).

6) Quais características as informações que o Sr.(a) usa para tomada de decisão, possuem hoje, na empresa?

( ) Facilidade de uso ( ) Qualidade ( ) Velocidade

( ) Precisão (ajuda o usuário a encontrar exatamente o que precisa em meio a um leque de opções)

( ) Adaptabilidade (é fácil de adaptar às minhas necessidades)

( ) Nenhuma das alternativas.

6.1) O que pode melhorar para que possa adotar decisões mais assertivas?

7) O Sr.(a) busca informações acerca da concorrência (estratégias que estão sendo usadas em outras instituições) para tomar suas decisões?

( ) Sim ( ) Não. Quais?

\begin{tabular}{|c|c|c|c|c|c|}
\hline (C) RDBCI: Rev. Digit. Bibliotecon. Cienc. Inf. & Campinas, SP & V.15 & n.1 & p. 53-81 & jan./abr. 2017 \\
\hline
\end{tabular}


8) Há na organização um grupo de pessoas responsáveis por gerir todas as todas as informações?

( ) Sim （ ） Não. Quem são esses profissionais?

9) Quais suportes tecnológicos o Sr.(a) usa para acessar informações?
( ) Meu celular
( ) Computador da instituição
( ) Internet da instituição
( ) Intranet
( ) Sistema Faciplac ( ) Outros:

10) O Sr.(a) recebeu treinamento para usar os suportes tecnológicos para acessar informação necessária à execução de suas atividades?

( ) Sim ( ) Não. Como foi o treinamento?

11) Quando vai tomar uma decisão quais os passos que segue?

12) Após a tomada de decisão o Sr.(a) utiliza algum processo de avaliação de eficiência, efetividade ou eficácia da decisão?

13) Sugere algo para melhorar a eficiência, efetividade, eficácia para avaliar as decisões?

\section{APÊNDICE C}

\section{TERMO DE AUTORIZAÇÃO DE USO DE IMAGEM E VOZ}

Neste ato, e para todos os fins em direito admitidos, autorizo expressamente a utilização da minha imagem e voz, em caráter definitivo e gratuito, constante em fotos, filmagens ou gravação de áudio decorrentes da minha participação na pesquisa de mestrado sobre - Gestão da informação para tomada de decisão realizada por Gleiciane Rosa da Silva, tendo como orientadora a professora Doutora Lillian Maria Araújo de Rezende Alvares, para o Programa de Pós-Graduação em Ciência da Informação (PPGCInf) da Faculdade de Ciência da Informação (FCI) da Universidade de Brasília (Unb).

As imagens e a voz poderão ser exibidas: nos relatórios parcial e final do referido estudo, na apresentação áudio-visual do mesmo, em publicações e divulgações acadêmicas, assim como disponibilizadas no banco de imagens resultante da pesquisa e na Internet. 
A aluna fica autorizada a usar imagens, filmagens e gravação de áudios, respeitando sempre os fins aqui estipulados.

Por ser esta a expressão de minha vontade, nada terei a reclamar a título de direitos conexos a minha imagem e voz ou qualquer outro.

Brasília, de de 2016.

\section{Assinatura}

Nome:

RG.: CPF:

Telefone1: ( ) Telefone2: ( ) 